\title{
Surgeon-controlled robotic partial nephrectomy for a rare renal epithelioid angiomyolipoma using near-infrared fluorescence imaging using indocyanine green dye: A case report and literature review
}

\author{
Timil H. Patel, BA; ${ }^{*}$ S. Joseph Sirintrapun, MD; ${ }^{*}$ Ashok K. Hemal, MD ${ }^{+}$ \\ *Wake Forest University School of Medicine, Medical Center Boulevard, Winston-Salem, North Carolina; †Director, Robotics and Minimally Invasive Surgery, Department of Urology, Wake Forest University \\ School of Medicine, Medical Center Boulevard, Winston-Salem, North Carolina
}

Cite as: Can Urol Assoc J 2012;6(2):e91-94. http://dx.doi.org/10.5489/cuaj.11250

\begin{abstract}
Renal epithelioid angiomyolipoma (E-AML) is a rare variant of angiomyolipoma (AML). It is a mesenchymal tumour believed to originate from the perivascular epithelioid cell (PEC). Unlike conventional $A M L$ which are benign, $\mathrm{E}-\mathrm{AML}$ has a rare aggressive behaviour. Conventional AML is typically triphasic containing adipose tissue, smooth muscle and dystrophic vessels in variable proportions, while E-AML are generally composed of plump spindled and polygonal-shaped "epithelioid cells" showing clear or eosinophilic cytoplasm and occasional pleomorphic multinucleated giant cells. E-AML can be misdiagnosed as renal cell carcinoma (RCC) when these "epithelioid cells" show clearing. Only a small number of cases of E-AML have been reported with the standard treatment being radical or partial nephrectomy. We report the first case report of a surgeon-controlled robotic partial nephrectomy using a nearinfrared fluorescence imaging using indocyanine green dye on a 25 -year-old woman with a T1B $(6.6 \mathrm{~cm})$ right renal mass. The final pathology revealed the diagnosis of E-AML. There was no recurrence and metastases after the 6-month follow-up.
\end{abstract}

\section{Case presentation}

A 25-year-old female, with no serious medical history, presented to the urology clinic to seek treatment options for a right renal mass. A few months ago, the patient was on a trip where she began to develop severe right flank pain, nausea and emesis. She was subsequently admitted to a hospital and underwent a computed tomography (CT) of the abdomen, which demonstrated a $6.5-\mathrm{cm}$ right renal mass with perinephric hemorrhage. She remained hospitalized for 3 days and was discharged for observation with a tentative diagnosis of a spontaneous hemorrhage from angiomyolipoma (AML). In clinic, the patient had no associated trauma or any lower urinary tract symptoms. She also denied any pain, gross hematuria, fever, chills or nausea. The patient had no history of recurrent urinary tract infections (UTI) or kidney stones. She consumes 5 alcoholic drinks per week, and had never smoked or used any drugs. Her immediate family did not have any serious medical conditions, although her uncle had renal cancer.

A repeat $\mathrm{CT}$ scan was done showing a persistent mass, measuring $5.9 \times 5 \times 5 \mathrm{~cm}$ (Fig. 1) exophytic fat containing mass arising from the lower anterior pole of the right kidney consistent with an angiomyolipoma. The mass appeared to be recruiting vessels from the retroperitoneum with the inferior portion of the mass draining into the right gonadal vein.

There was a long and thorough discussion with the patient about the disease, pathophysiology and management of AML. Due to the size of the lesion and risk of future hemorrhagic events, a long-term observation was not recommended. She was presented with several treatment options: selective angiographic embolization, an attempt at surgeoncontrolled robotic partial nephrectomy (SCRPN) or open partial or right radical nephrectomy $(\mathrm{RN})$. She sought several opinions and finally decided to go ahead with SCRPN at our centre.

The patient underwent a transperitoneal right-sided SCRPN (Fig. 2a). ${ }^{1,2}$ A total of 4 robotic ports (one for a 12-mm camera, three additional $8-\mathrm{mm}$ robotic ports) and one 12-mm assistant port were placed. After dissecting the renal vessels and tumour, renal ultrasound was performed and subsequently 2 cc indocyanine green (ICG) dye was injected prior to near-infrared (NIR) imaging to identify renal vessels and for imaging differentiated renal parenchyma and mass (Fig. 2b). Prior to clamping the renal artery, a $12.5 \mathrm{mg}$ of mannitol and $20 \mathrm{mg}$ of lasix was administered. Under near-infrared fluorescence imaging using indocyanine green dye, the renal artery was clamped with a surgeon-controlled bull dog clamp and the renal mass was excised maintaining normal renal parenchyma all around (Fig. 3a, Fig. 3b). Renorrhaphy was performed using unibarb suture. Total warm ischemia time was 7 minutes. Adequate hemosta- 


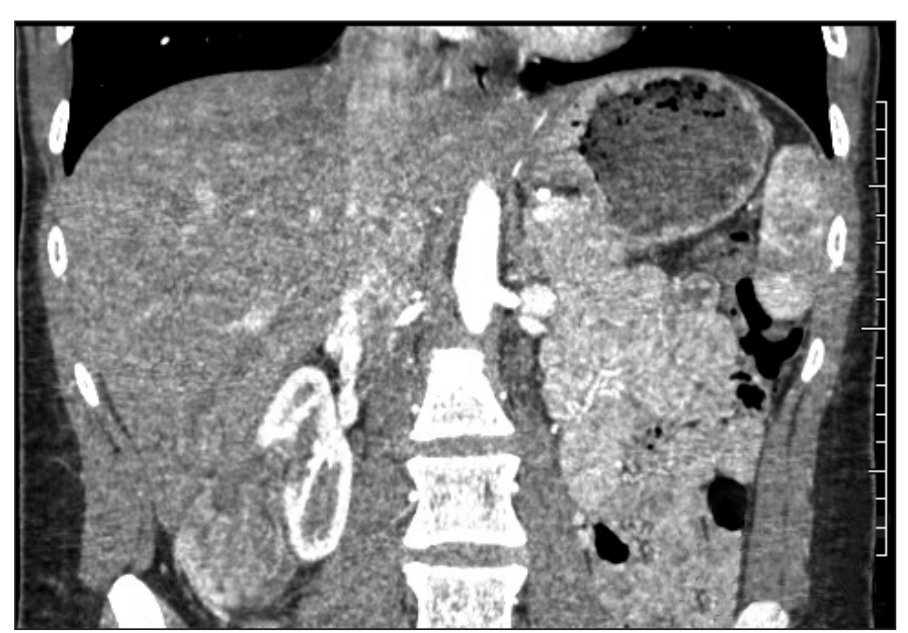

Fig. 1. Computed tomography of the abdomen showing preoperative exophytic fat containing mass arising from the lower pole of the right kidney consistent with an angiomyolipoma.

sis was ensured and the specimen was entrapped with a Endocatch I (Ethicon Endo-Surgery, Inc., Cincinnati, $\mathrm{OH}$ ) and removed. There were no apparent complications and the estimated blood loss was $75 \mathrm{~mL}$. Her postoperative recovery was unremarkable and she was discharged in less than 48 hours.

\section{Pathological evaluation}

Sections showed a tumour with mature adipose tissue alternating with areas of necrosis and hemorrhage (Fig. 4). The non-adipocytic areas of the tumour were composed of "epithelioid" polygonal clear and granular eosinophilic cells (Fig. 5). Scattered dysmorphic vessels were present and mitotic index is low (1 per 10 high power fields). Atypical mitoses, cellular anaplasia, pleomorphic giant cells and lymphovascular invasion were absent. Immunohistochemistry

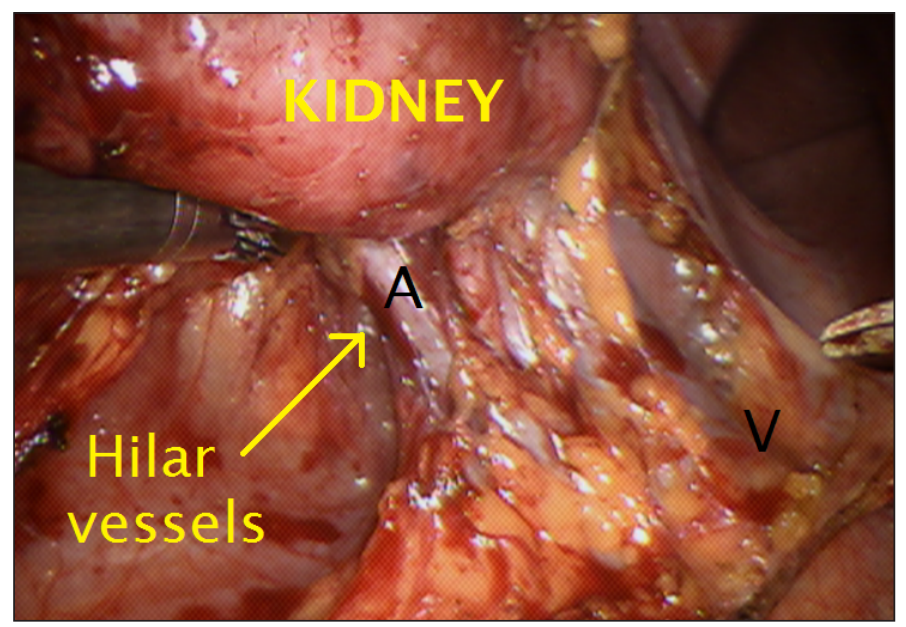

Fig. 2a. Demonstrates kidney and dissected hilar vessels (patient had 1 renal artery and 2 renal veins) for preparation of clamping the renal artery. showed no staining for pancytokeratin AE1/3, excluding the morphologic differential of clear cell renal cell carcinoma (RCC). HMB-45 (human melanoma, black) positive staining confirmed the morphologic impression of angiomyolipoma. The percentage of mature adipose tissue in the tumour is about $60 \%$, with the remainder of tumour comprising of mostly "epithelioid" cells with interspersed dysmorphic vessels, solidifying the diagnosis of E-AML.

\section{Discussion}

In this patient, we used surgeon-controlled bull dog clamp, ultrasound and ICG dye for NIR imaging to excise the tumour (T1b), while maintaining normal renal parenchyma all around. This technique allowed surgeons to see in realtime imaging, while making actual incision into the renal parenchyma. A partial nephrectomy is unquestionably the favourable procedure as it aims to preserve the patient's kidney. We believe performing a surgeon-controlled robotic partial nephrectomy is an excellent alternative to traditional open or laparoscopic partial nephrectomy in the treatment of epithelioid angiomyolipoma (E-AML). Surgeon-controlled robotic surgery using the da Vinci Si Surgical System (Intuitive Surgical, Sunnydale, CA) with ICG and NRI imaging further helps in undertaking SCRPN. A 6-month follow-up with imaging study did not show recurrence (Fig. 6).

Renal AML is a mesenchymal tumour comprised of dysmorphic blood vessels, adipose tissue and smooth muscle. ${ }^{3}$ AML is generally described as benign, and patients most often never show recurrence or metastasis on clinical follow-up. ${ }^{3}$ The histological diagnosis of conventional AML is fairly standard with typical cases containing adipose tissue, smooth muscle and thick-walled vessels. ${ }^{4}$ The diagnosis

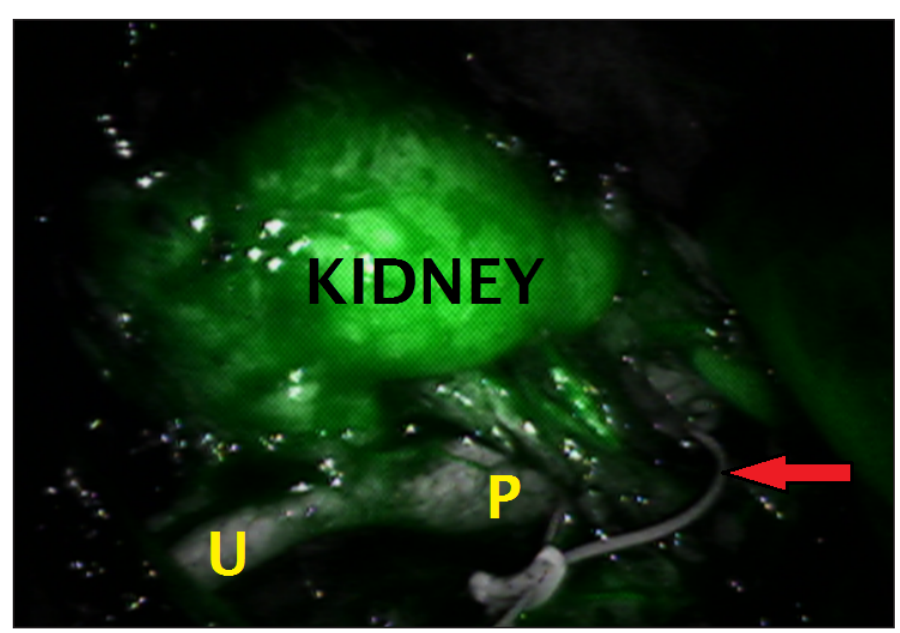

Fig. $\mathbf{2 b}$. Indocyanine green dye was injected prior to near-infrared imaging to identify renal vessels and for imaging differentiated renal parenchyma and mass. The kidney is illuminated bright green. The angiomyolipoma is black. The renal artery (red arrow), renal pelvis (P) and ureter (U) can also easily be identified. 


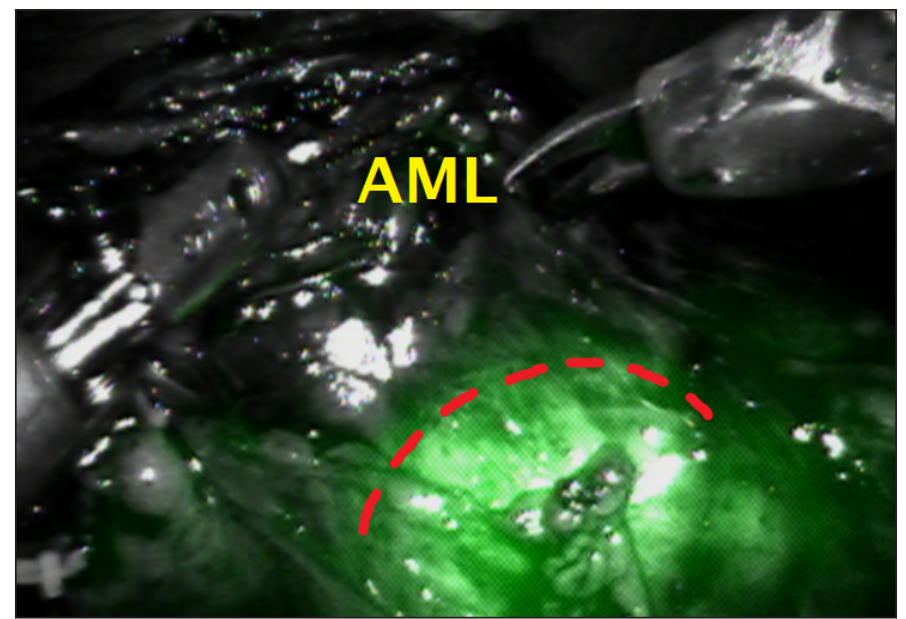

Fig. 3a. Surgeon-controlled partial nephrectomy in process. The red-line approximately demonstrates the margin of excision.

becomes challenging, however, when one of these components becomes markedly prevalent and overtaking the other components. This, in turn, can mimic other benign and malignant tumours. ${ }^{4}$ For instance, a conventional AML, which is predominantly smooth muscle, can mimic a benign leiomyoma. If this smooth muscle-rich AML were to have numerous mitoses or cytologic atypia, as conventional $\mathrm{AML}$ can show, leiomyosarcoma enters the differential. Epithelioid angiomyolipoma (E-AML) is a rare variant of AML that unlike conventional $\mathrm{AML}$, has a reported malignant propensity for metastasis, particularly to lymph nodes, lung, liver, or spine. ${ }^{5}$

$\mathrm{E}-\mathrm{AML}$ is extremely rare with an incidence of $0.22 \%$ for women and $0.1 \%$ for men, according to an ultrasound based study of 17941 health individuals. ${ }^{6}$ The mean age at diagnosis is 38 years. ${ }^{5}$ Interestingly, more than half of the recorded patients of EML also suffer from tuberous sclerosis. ${ }^{5} \mathrm{E}-\mathrm{AML}$ occurs primarily in the kidneys, but it has been shown to transpire also in the liver, pancreases, pelvic cavity and within osseous structures. ${ }^{7}$ E-AML tumours are comprised of round to polygonal epithelioid cells with clear or granular eosinophilic cytoplasm intermixed with spindle cells, often showing varying degress of mitotic activity. 3,5

The epithelioid cells of E-AML can show significant pleomorphism. However, the appearance of a "standard" epithelioid cell is variable because some epithelioid cells can have small nuclei and small nucleoli with limited amounts of cytoplasm, while other epithelioid cells can have large eccentrically-placed vesicular pleomorphic nuclei and prominent macronucleoli and abundant cytoplasm. ${ }^{5}$ Hemorrhage, necrosis, vascular invasion and extention into perirenal soft tissues is also common in many cases. ${ }^{3,5}$ The presence of giant multinucleated cells, is common in E-AML. ${ }^{6}$ By contrast, giant multinucleated cells are rarely reported in clear cell RCC, the morphologic differential of E-AML. ${ }^{6}$ The pathologic features that predict adverse prognosis in E-AML are poorly defined due to the rarity of tumour,

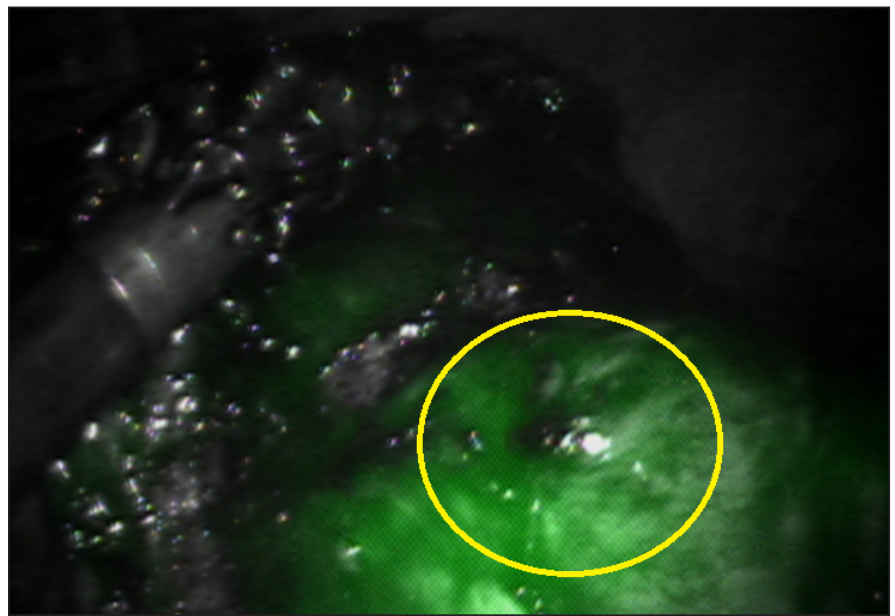

Fig. 3b. Excision of angiomyolipoma in progress. Yellow circle demarcates the base of normal renal parenchyma.

difficulty for pathologists to recognize and define E-AML and lack of prolonged information and follow-up.

Immunochemistry provides essential information for a differential diagnosis of E-AML. E-AML stains strongly for melanoma-associated markers, particularly HMB-45 (human melanoma, black). ${ }^{8}$ Because E-AML can show polygonal cells with clearing, it can often be misdiagnosed as clear cell RCC. Careful morphologic examination along with HMB-45 positive staining and negative staining for epithelial markers, like pancytokeratin, can help to successfully differentiate E-AML from clear cell RCC or other primary

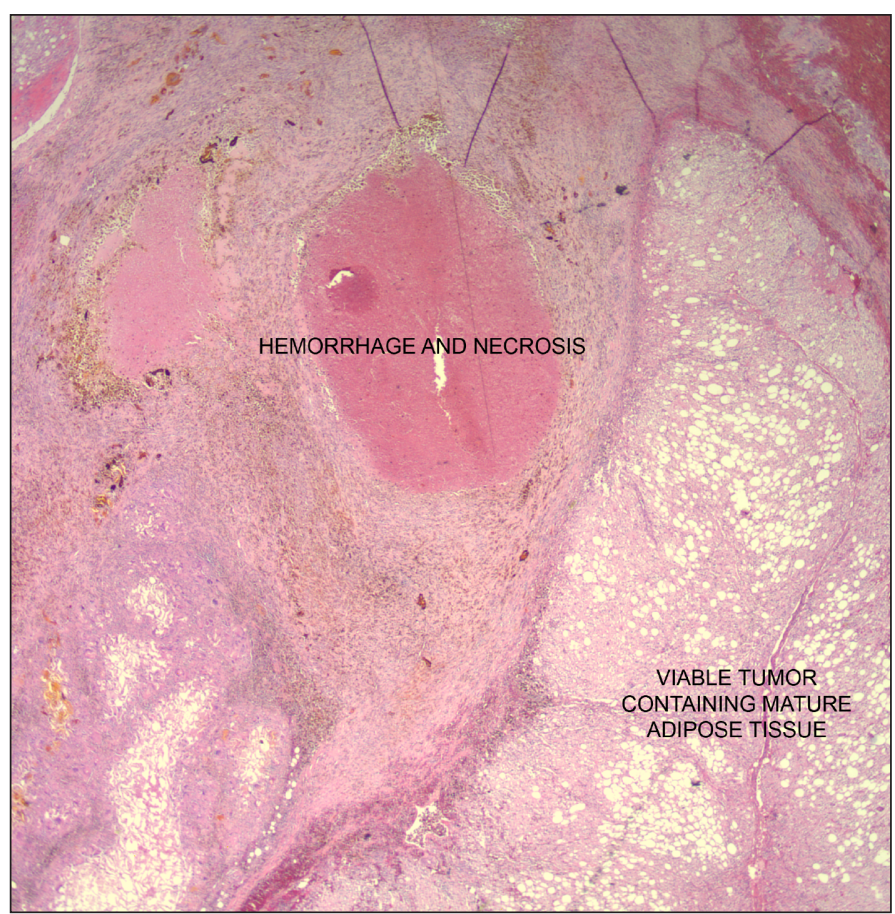

Fig. 4. Viable tumour containing mature adipocytic regions alternating with areas of hemorrhage and necrosis at $20 x$. 
Patel et al.

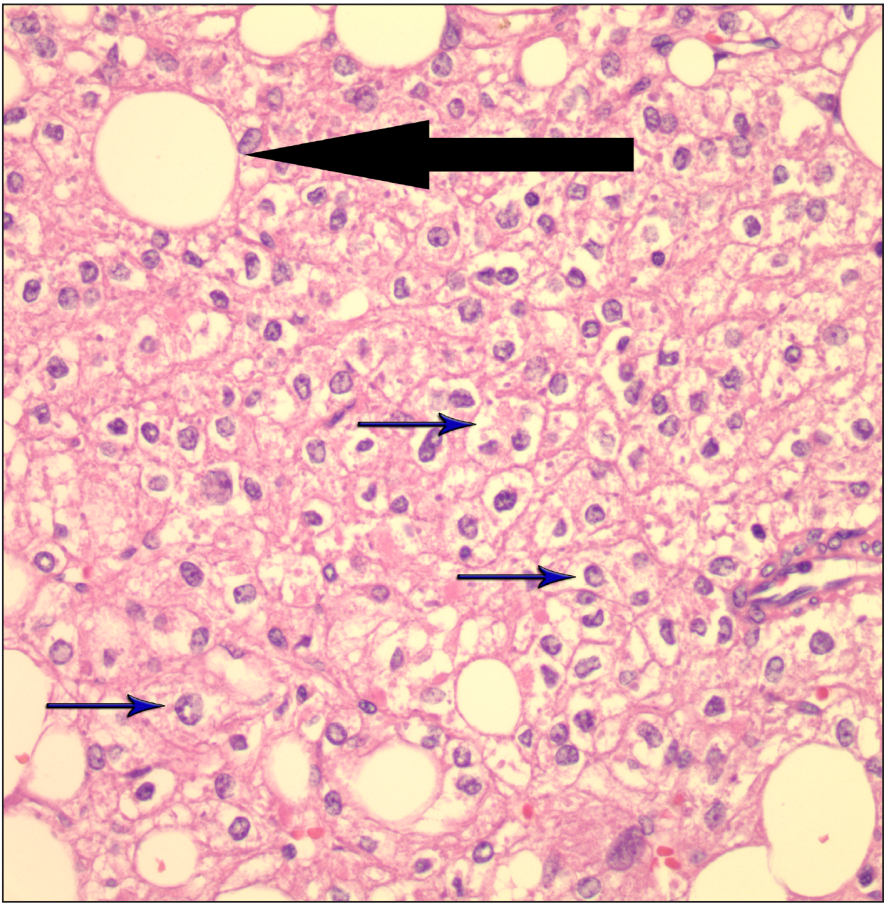

Fig. 5. Polygonal "epithelioid" clear cells; some with granular eosinophilic cytoplasm (small blue arrows) interspersed with mature adipocytes (large solid black arrows) at $200 x$

renal neoplasms, which do not stain for HMB-45 and are positive for epithelial markers. ${ }^{8}$

\section{Conclusion}

E-AML has a slow growth and infiltration rate, resulting in generally no specific clinical symptoms when the tumour is small. ${ }^{7}$ For larger tumours, however, surgical excision is the mainstay treatment. ${ }^{7}$

Competing interests: None declared.

This paper has been peer-reviewed.

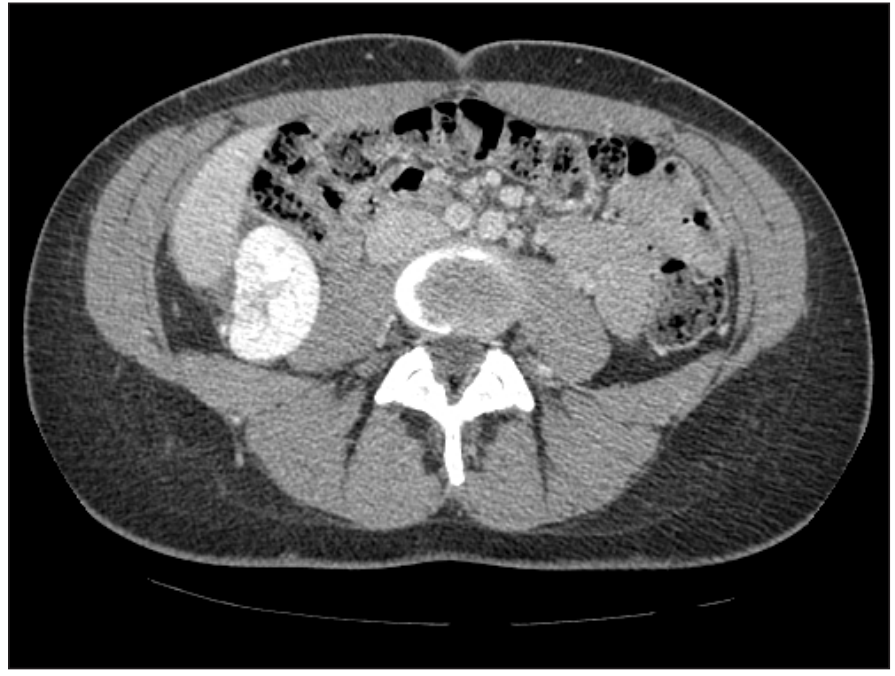

Fig. 6. Computed tomography of the abdomen showing postoperative changes of surgeon-controlled robotic partial nephrectomy with no complicating features evident.

\section{References}

1. Babbar P, Hemal AK. Robot-assisted partial nephrectomy: current status, techniques, and future directions. Int Urol Nephrol 2012;44:99-109. Epub 2011 Feb 25. http://dx.doi.org/10.1007/s11255-0119900-6

2. Sukumar S, Rogers CG. Robot-assisted partial nephrectomy. J Endourol 2011;25:151-7. http://dx.doi. org/10.1089/end.2010.0672

3. Bing Z, MacLennan G. Renal Epithelioid Angiomyolipoma. J Urol 2009;182:2468-9. http://dx.doi. org/10.1016/i.juro.2009.08.082

4. Svec A, Velenska Z. Renal epithelioid angiomyolipoma-a close mimic of renal cell carcinoma. Report of a case and review of the literature. Pathol Res Pract 2005;200:851-6. http://dx.doi.org/10.1016/i. prp.2004.09.004

5. Bostwick DG, Cheng L. Urologic Surgical Pathology. Philadelphia, PA: Elsevier; 2011:130-4.

6. Fuji Y, Aijma Jl, Oka K, et al. Benign renal tumors detected among healthy adults by abdominal ultrasonography. Eur Urol 1995;27:124-7.

7. Cui L, Xiao H, Shen-Chu G, et al. A massive renal epithelioid angiomyolipoma with multiple metastatic Iymph nodes. Clin Imaging 2010;35:320-3. http://dx.doi.org/10.1016/j.clinimag.2010.08.017

8. Wen J, Li HZ, Ji ZG, et al. Renal epithelioid angiomyolipoma without obvious local progress in 10 years: a case report and literature review. Ir J Med Sci 2011;180:557-60. http://dx.doi.org/10.1007/ s1 1845-010-0616-x

Correspondence: Dr. Ashok K. Hemal, Professor, Department of Urology and Institute of Regenerative Medicine, Director, Robotics and Minimally Invasive Surgery, Wake Forest University School of Medicine, Department of Urology, Medical Center Boulevard, Winston-Salem, NC 27157; fax: 336-716-5711; ahemal@wfubmc.edu 